\title{
Estrés laboral y desarrollo de trastornos psicosomáticos en estudiantes de posgrado
}

\section{Workplace stress and development of psychosomatic disturbances in graduate students}

\author{
José Raúl Peralta López y Walter Michael Villalba Jiménez ${ }^{l}$ \\ Citación: Peralta L., J.R. y Villalba J., W.M. (2019). Estrés laboral y desarrollo de trastornos \\ psicosomáticos en estudiantes de posgrado. Psicología y Salud, 29(2), 177-186.
}

RESUMEN

El presente estudio correlacional tuvo como objetivo identificar los niveles de estrés laboral que estudiantes de posgrado pueden manifestar ante la sobrecarga de trabajo. Los resultados mostraron una relación significativa entre el desarrollo del estrés y el desencadenamiento de dichos trastornos según la Escala de Desgaste Ocupacional. A partir de estos resultados, se propone contar con especialistas en salud mental en los centros educativos que ofrecen estudios de posgrado a fin de atender este tipo de afectaciones, contribuyendo así a mejorar la calidad de vida de los estudiantes.

Palabras clave: Estrés laboral; Trastornos psicosomáticos; Sobrecarga; Estudiantes de posgrado.

\begin{abstract}
The aim of this correlational study was to identify the stress levels of graduate students due to work overload. Results revealed a significant relationship between stress levels and events triggering such stress disorders, as measured by the Occupational Wear Scale. On the basis of these results, it is proposed that mental health specialists attend this type of affectations within the educational centers that offer graduate degrees, in order to contribute to improving students' quality of life.
\end{abstract}

Key words: Work stress; Psychosomatic disorders; Work overload; Graduate students.

\section{INTRODUCCIÓN}

ऍy,c

as demandas y exigencias que se han generado dentro del campo laboral han hecho que los profesionistas deban capacitarse constantemente a fin de otorgar mejores servicios a quienes los requieran y, con ello, lograr la calidad de su propio centro de trabajo. Sin duda, el sistema educativo es parte esencial y primordial en este proceso, de modo que ha llevado a los profesionales de la educación a una permanente actualización para que respondan a las nuevas tendencias que se van forjando en el mundo actual. Son los estudios de posgrado una opción para lograr este fin y así cubrir las necesidades que la propia sociedad demanda. La Estrategia Nacional de Formación Continua de Profesores de Educación Básica y Media Superior de la Secretaría de Educación Pública (SEP) (2016) da cuenta de la concepción actual del docente como un gestor axiológico cultural de las transformaciones sociales derivadas de las exigencias

${ }^{1}$ Centro de Estudios Especializados Ausubel, Hacienda Sta. Rosa Num. 13, La Hacienda, 91630 Jacarandas, Ver., tel (228)117-86-56, correos electrónicos: ceea24@hotmail.com y peraltalopez_62@hotmail.com. Artículo recibido el 4 de agosto y aceptado el 30 de octubre de 2018. 
de esta nueva sociedad del conocimiento, misma que ha incidido en las universidades y en todos sus miembros (Hernández, 2018).

Expone Andere (2012): "Los maestros del siglo XXI no son instructores, no están todo el tiempo frente a un grupo y no son sumisos; son educólogos, multifuncionales y profesionales". Según dicha acepción, los docentes adquieren un compromiso ante la sociedad que los lleva a tomar la decisión de actualizarse mediante los estudios de posgrado, y solventar con ello las actuales necesidades del contexto laboral. No obstante, esta elección genera incertidumbre, pues ingresar a estos programas compromete al aspirante en virtud de que las exigencias derivadas de las actividades y tareas que debe desempeñar son aún mayores, lo que propicia el desencadenamiento del estrés (Barraza, 2008), mismo que puede afectar su estado de salud.

En efecto, la multiplicidad de tareas que debe atender el estudiante de posgrado puede producir estrés laboral o académico (Berrio y Mazo, 2011) debido a las actividades que su centro de trabajo demanda y a la vez las que sus estudios implican, lo que origina una sobrecarga laboral que se complica aún más por las responsabilidades familares, lo que hace todavía más compleja su situación.

De allí el interés de los estudiosos de la conducta humana por desarrollar investigaciones sobre el estrés laboral y académico, evaluar su impacto en el estado de salud física o mental de quienes lo padecen e identificar las consecuencias que puede acarrear este proceso, que puede afectar de manera considerable el estilo de vida de las personas (Naranjo, 2009), para así generar estrategias para afrontarlas de una mejor manera. Berrio y Mazo (2011) señalan que una consecuencia que puede derivarse del estrés es el desarrollo de diversas patologías que afectan drásticamentemente la salud.

Tomando en cuenta los planteamientos anteriores, pareció necesario integrar en la presente investigación dichos elementos, parte de la vida cotidiana del estudiante de posgrado por la diversidad de tareas laborales, académicas y familiares que debe atender. Así, se aborda de una manera general el concepto de estrés y las variables que entraña, a fin de asociarlo con los trastornos psicosomáticos que pueden incidir de manera signi- ficativa en el desarrollo de los propios estudiantes, y finalmente se describe el estudio realizado mediante la aplicación de la Escala de Desgaste Ocupacional de Uribe (2010).

\section{Conceptualización y antecedentes del estrés}

La palabra estrés deriva del inglés stress ("tensión"), término retomado de la física, cuyo significado proviene del griego stringere, un término que desde el siglo XIV se ha utilizado en diversos textos para referirse a aquellos sucesos que provocan tensión, pero fue hasta 1956 cuando Hans Selye, un fisiólogo y médico austrohúngaro-canadiense, lo llevó al campo de la salud, denominándolo "síndrome general de la adaptación” (cf. Selye, 1956), y proponiendo que varias enfermedades se generaban por un prolongado estrés en los órganos, como la hipertensión arterial o los problemas cardiacos. Mediante sus investigaciones, este autor identificó que había diversos factores, como las demandas sociales o ambientales, que pueden provocar estrés debido a una adaptación inapropiada ante esas circunstancias, provocando con ello el desarrollo de ciertas enfermedades fisiológicas (Berrio y Mazo, 2011).

Son tres las fases propuestas por Selye (1956) (cf. Naranjo, 2009); la primera, fase "aguda o alerta", se origina cuando una eventualidad o factor estresante provoca una reacción fisiológica, poniendo en alarma al organismo ante dicho suceso, el cual se espera que se supere rápidamente. Cuando eso no ocurre, se entra en la segunda fase, denominada "resistencia", cuya finalidad es resistir esa eventualidad; si no es posible solventarla o resolverla de manera inmediata, se genera frustración, que a su vez conduce a la última fase del estrés, "agotamiento", que puede provocar otras afectaciones psíquicas, como el desarrollo de ansiedad o depresión.

Con esas aportaciones, el estrés se ha vuelto un sujeto primordial de estudio en muchas y muy diversas investigaciones, pues se ha demostrado que provoca numerosas enfermedades psicológicas y fisiológicas en cuanto que involucra a todos los sistemas y subsistemas del organismo (Cólica, 2012), causando así afectaciones en la salud física y mental de quien lo padece. 


\section{Etiología del estrés}

Chiavenato (2009) apunta que al ocurrir una situación estresante el cuerpo empieza a segregar sustancias químicas que hacen que se generen ciertos síntomas en el organismo que provocan distintos cambios biológicos que detonan una elevada presión sanguínea, nerviosismo y tensión, así como preocupación crónica e incapacidad para relajarse, lo cual puede propiciar el consumo excesivo de tabaco y alcohol, insomnio, sentimientos de incapacidad, inestabilidad emocional y problemas digestivos, entre muchas otras alteraciones fisiológicas.

Al momento en que se genera esa alerta, ocurre neurológicamente una segregación de diversas sustancias que ocasionan cambios orgánicos que se reflejan en el comportamiento del individuo, pues al llegar este mensaje al cerebro se segrega noradrenalina, que provoca a su vez una hipervigilancia ante el suceso que se está suscitando. Al aumentar dicha situación, la glándula suprarrenal libera adrenalina y, con ello, la aceleración del ritmo cardíaco, que se ve reflejado en la dilatación de los pulmones y vasos sanguíneos, respuesta que puede provocar el abandono de la situación problemática; sin embargo, cuando el estrés se prolonga, es posible que se activen ciertos mecanismos alostáticos y el eje hipotálamo-hipófisis-adrenal, segregando así hormonas como la corticotropina o la adrenocortitropina, lo que causa a su vez un desequilibrio bioquímico del propio organismo y provoca enfermedades que pueden llevar a la muerte de no atenderse (Herrera et al., 2017).

Dichos componentes bioquímicos pueden verse reflejados fisiológicamente (Naranjo, 2009) a través de un aumento de la sudoración corporal, sensación de estómago contraído, contracción de los músculos de los brazos y piernas o una mayor agitación causada por el aumento en la circulación del torrente sanguíneo, lo que produce una incapacidad para permanecer quieto.

\section{Estrés laboral y estrés académico}

Berrio y Mazo (2011) destacan dos categorías que pueden causar el desarrollo del estrés: académica y laboral, cada una de ellas con diversas complejidades que dependen del contexto ambiental en que se susciten; así, mientras que el estrés laboral es originado por una sobrecarga en el propio trabajo, el académico lo es por las demandas escolares; pero ambos tipos de estrés ponen en juego la capacidad cognitiva y emocional de la persona para solventar las tareas que lleva a cabo y que la conducen al desgaste físico y mental.

La docencia se ha catalogado como una de las profesiones en las que una persona es más proclive a desarrollar estrés debido a la diversidad de actividades que debe cubrir, como atender los problemas de disciplina, la preparación de los cursos y las demás actividades que la institución educativa le demanda cotidianamente (E1 Sahili, 2011), situación que se vuelve más compleja cuando el docente estudia un posgrado.

Al ser la docencia una actividad multiocupacional, quien la ejerce es propenso a desarrollar los síntomas asociados al estrés laboral, provocando con ello el síndrome de burnout, que implica una sensación del fracaso y una existencia caracterizada por el agotamiento. Reyes, Ibarra, Torres y Razo (2012) refieren que todo ello puede provocar la desmotivación ante la propia profesión, y asimismo destacan las dimensiones propuestas por Maslach (1978) y Maslach y Jackson (1982) que propician esta sintomatología: el agotamiento emocional, la despersonalización y la baja autoestima. El primero está relacionado con el cansancio físico y emocional debido a una jornada de trabajo complicada; la segunda trae como consecuencia sentimientos de desesperanza personal, indefensión e impotencia, de modo que se generan en la persona actitudes negativas hacia las actividades laborales o académicas, lo que tiene como resultado un comportamiento apático, y la tercera, la baja autoestima, se traduce en una pérdida de ideales, en un alejamiento de las actividades familiares, sociales y recreativas y en una incapacidad de la persona para sentirse productiva y autorrealizada.

Ciertos sucesos pueden generar más estrés en algunas personas que en otras en virtud de a las variables contextuales propias del ámbito del individuo, las variables personales del mismo y las propias consecuencias del estrés (Ayuso, 2016), por lo que el evento puede ser calificado desde diversas perspectivas según los rasgos individuales de la persona, de modo que distintas personas responden de modos diferentes ante una misma eventualidad. 
El estrés puede agudizarse cuando la persona decide incursionar en estudios de posgrado, propiciando así el desarrollo del estrés académico, que es un proceso sistémico de carácter adaptativo y psicológico que suele aparecer cuando se está sometido a contextos escolares que demandan continuamente el cumplimiento de tareas y actividades académicas (Toribio y Franco, 2016). Pulido et al. (2011) destacan que "el estrés académico aumenta conforme el estudiante progresa en sus estudios" (p. 32). Tales exigencias personales se presentan más en aquellos que estudian posgrados, pues en ocasiones, al considerárseles un ejemplo a seguir, tratan de comprometerse cada vez más con las encomiendas de los catedráticos, y si bien obtienen así un mayor peso moral y ético entre quienes los rodean, el costo es un mayor estrés.

En consecuencia, el estrés académico puede considerarse como un elemento que favorece el deterioro de la salud mental (Suárez y Díaz, 2015); no atender a tiempo los síntomas que provoca puede conducir a un padecimiento mayor, y con ello verse afectada severamente la salud del estudiante.

De allí la necesidad de que las instituciones que ofrecen estudios de posgrado desarrollen estrategias de prevención, promoción e intervención para el manejo del estrés (Mazo, Londoño y Gutiérrez, 2013) con la finalidad de lograr una formación integral que, además de propiciar el desarrollo de conocimientos, coadyuve a la conservación o mejora de la salud mental de quienes participan en ellas, de modo que aprendan a manejar adecuadamente las situaciones estresantes que perjudican el desarrollo psíquico.

\section{Factores psicosomáticos asociados al estrés}

Viaco y Abello (2014) refieren que ante las exigencias laborales y ocupacionales se pueden generar factores de riesgo psicosocial que alteran considerablemente el estado de salud; en efecto, las demandas cognitivas, psicológicas y emocionales que se requieren para el desarrollo y cumplimiento de diversas tareas constituyen el elemento primordial para el desarrollo de diversas sintomatologías. Aunque al comienzo de las investigaciones se creía que era algo exclusivo de las organizaciones laborales, la propia Organización Mundial de la
Salud ha reconocido que otros factores ambientes pueden originar la aparición de tales patologías asociadas con el estrés.

Uribe (2010), distingue siete factores psicosomáticos vinculados directamente con el estrés, a saber: a) trastornos del sueño, b) dolor, c) ansiedad, d) trastornos neuróticos, e) trastornos gástricos, f) trastornos psicosexuales y g) depresión.

a) El sueño, un elemento primordial para el desarrollo biológico y psicológico del ser humano, puede verse afectado cuando se experimentan situaciones estresantes, lo que propicia alteraciones que originan trastornos como el insomnio, el más común, que afecta las relaciones interpersonales debido a los cambios de humor que origina en la persona al no tener un descanso adecuado y saludable (Portilla, 2006).

b) Otro de los factores psicosomáticos asociados con el estrés es el dolor. En las últimas décadas diversos investigadores han identificado que el estrés puede amplificar el dolor o, por el contrario, el dolor puede verse como un estresor; sin embargo, en ambos casos suele aparecer en las personas con mayor sensibilidad y activación, o con un menor umbral de reactividad ante los estímulos, desarrollando así en ellas una comorbilidad psicopatológica asociada con determinados agentes bioquímicos o ciertas hormonas secretadas ante las emociones negativas, causando con ello afectaciones fisiológicas (Sánchez, Sirera, Peiró y Palmero, 2008) -como dolor de cabeza o espalda, por ejemplo- que afectan el cumplimiento de las actividades al sentirse incompetentes para realizarlas debido a tal sintomatología.

c) A la ansiedad, considerada un trastorno mental, se le ha relacionado asimismo con el estrés. Según Zúñiga (2014), este trastorno aparece cuando se llevan a cabo tareas o actividades que demandan una mayor atención, lo que provoca así una afectación o alteración que la convierte en una patología al aumentar la frecuencia de ciertos síntomas, como irritabilidad general, angustia, terrores nocturnos y vértigos.

d) Se consideraba que al principio de los estudios freudianos se asociaban los problemas de ansiedad con los trastornos neuróticos o "neurastenia", pero desde 1980 la propia Asociación Americana de Psicología ha sustituido este último término por el de "trastornos de ansiedad"; no obstante, 
es todavía un término permitido, sobre todo por la Organización Mundial de la Salud, que retoma las características propuestas por George Beard en 1880: estado crónico de agotamiento, corazón irritable, síntomas generalizados de incomodidad y disfunciones corporales (cf. también Sarudiansky, 2013), síntomas que pueden generarse por el estrés.

e) Los trastornos gastrointestinales son considerados como uno de los factores psicosociales con mayor relevancia en aquellas personas que sufren estrés continuamente. Como apuntan Montaño, Dossman, Herrera, Bronet y Moreno (2006), la mayor parte de las reacciones ante situaciones estresantes se concentran en el aparato gastrointestinal, causando con ello malestares estomacales persistentes y diversas enfermedades fisiológicas, como la gastritis, un padecimiento común en aquellos individuos que viven en ambientes estresantes, el cual, si no es atendido de manera adecuada, puede provocar el desarrollo de úlceras gástricas y la proliferación de la bacteria Helicobacter pylori. De allí la necesidad de dar una atención temprana y oportuna a la persona cuando muestra síntomas asociados a esa patología.

f) Si bien el sexo es considerado como un elemento clave para reducir los grados de estrés, este último puede afectar la vida sexual cuando es prolongado, causando con ello afectaciones en el apetito sexual que generan a la postre disfunciones de la propia sexualidad (Gómez, 2014).

g) Las exigencias laborales y académicas pueden orillar a que los estudiantes generen sentimientos de inferioridad, y en consecuencia depresión, lo que provoca que con frecuencia decidan desertar de los estudios de posgrado -o en casos muy extremos llegar al suicidio-, al sentirse incompetentes para satisfacer las exigencias del programa (Mayor, Coral y Ríos, 2009).

Como se puede apreciar, el estrés puede provocar diversos trastornos y afectar considerablemente la vida de quien lo padece al producir alteraciones en el desarrollo psíquico y causar daños a los procesos biológicos. De allí que el presente estudio correlacional, toda vez que se asocian correlativamente variables mediante un patrón predecible para un grupo o población (Hernández, Fernández y Baptista, 2010), tuviera como propósito identificar la relación que existe entre el estrés y el desencadenamiento de los factores psicosomáticos en una muestra de estudiantes de posgrado, así como analizar si quienes mantienen una relación de pareja son más afectados por esta variable, o bien los que carecen de ella.

\section{MÉTODO}

\section{Participantes}

Participaron 63 estudiantes de posgrado de un centro de estudios especializados del municipio de Emiliano Zapata en el estado de Veracruz (México). De ellos, 36 mantenían una relación conyugal. En su mayoría, tenían uno o más trabajos, motivo por el cual habían decidido limitar sus estudios a los fines de semana, generando con ello una carga adicional a sus tareas y actividades laborales. La muestra se determinó teniendo como criterio que los estudiantes cursaran el cuarto semestre de la Maestría en Educación, ya que en este semestre, además de las actividades convencionales que implica el posgrado, se añade la elaboración de la tesis, misma que debe presentarse al concluir dichos estudios, lo que implica un aumento considerable del grado de estrés y el desarrollo de ciertos trastornos psicosomáticos.

\section{Instrumento}

Escala de Desgaste Ocupacional (Uribe, 2010).

La Escala de Desgaste Ocupacional fue el instrumento que se utilizó para identificar el grado de estrés en los estudiantes de posgrado, el cual se retoma de los modelos de Leiter y Maslach (1988) y Golembewsky y Muzenrider (1984). Dicha escala, que tiene una confiabilidad de 91\%, medida por el coeficiente alfa de Cronbach, fue elaborada para identificar el grado de estrés laboral y las enfermedades psicosomáticas que pueden desencadenarse debidas al mismo. Este instrumento consta de 105 ítems, 70 de los cuales están diseñados para conocer si la persona está o no de acuerdo con las afirmaciones que contiene para, a partir de allí, identificar el grado de estrés o el desarrollo de algún factor psicosomático. Los primeros 30 ítems valoran el estrés, mientras que los 40 restantes se enfocan en la identificación de esos factores 
psicosomáticos. Los ítems del 71 al 105 permiten conocer las características generales de la persona, como el estado civil, edad, sexo, escolaridad y otros elementos que hacen posible determinar un diagnóstico.

\section{RESULTADOS}

Como se puede observar en la Tabla 1, la mayoría de los estudiantes estaban casados, mientras que el resto señaló no tener una relación de pareja, por lo que podían dedicarse más a sus estudios. Es importante señalar que la sobrecarga de trabajo puede limitar de manera considerable la convivencia familiar y ser un desencadenante potencial de problemas en la relación conyugal, convirtiéndose así en un estresor crónico (Berumen y González, 2011). En la población de estudio se manifestó tal estrés en $14.2 \%$ de los participantes en cuanto a su estado civil.

Tabla 1. Estado civil de la muestra.

\begin{tabular}{|c|l|c|r|r|c|}
\cline { 3 - 6 } \multicolumn{2}{c|}{} & Frec. & \multicolumn{1}{c|}{$\%$} & $\begin{array}{c}\% \\
\text { válido }\end{array}$ & $\begin{array}{c}\% \\
\text { acumulado }\end{array}$ \\
\hline \multirow{3}{*}{ Válido } & Casados & 36 & 57.1 & 57.1 & 57.1 \\
\cline { 2 - 6 } & Solteros & 27 & 42.9 & 42.9 & 100.0 \\
\cline { 2 - 6 } & Total & 63 & 100.0 & 100.0 & \multicolumn{1}{|c}{} \\
\hline
\end{tabular}

En el manual de Uribe (2010), el desgaste ocupacional se clasifica en tres niveles: agotamiento, despersonalización e insatisfacción de logro, en cada uno de los cuales se distinguen cinco grados de desarrollo: bajo, abajo del término medio, arriba del término medio, alto y muy alto, lo que permite calificar la afectación que la sobrecarga laboral genera en la persona y provoca con ello alteraciones en su estado de salud.

En la Tabla 2, que alude al desgaste ocupacional, se muestra la afectación generada por la sobrecarga laboral; en la misma se hace una comparación entre los estudiantes que mantenían una relación de pareja y los que carecían de ella, observándose que los primeros manifestaban un mayor agotamiento, por arriba del término medio, lo que puede asociarse a otras actividades a desempeñar distintas a las laborales y académicas. En el aspecto “despersonalización”, son pocos los casos con índices por arriba de lo normal, aunque en $1.6 \%$ de los estudiantes casados apareció este factor en un alto grado; por último, en "insatisfacción de logro" se obtuvo un registro de $7.9 \%$ con alto grado en ambos casos, sin embargo, 1.6\% de los estudiantes que no tenían ninguna relación se ubicaron en este punto en la clasificación, lo que lleva a pensar que las tareas a las cuales estaban expuestos sobrepasaron su límite.

Tabla 2. Desgaste ocupacional.

\begin{tabular}{|c|c|c|c|c|c|c|c|}
\hline & \multicolumn{2}{|c|}{ Agotamiento } & \multicolumn{2}{|c|}{ Despersonalización } & \multicolumn{2}{|c|}{ Insatisfacción de logro } \\
\hline & & Solteros & Casados & Solteros & Casados & Solteros & Casados \\
\hline \multirow{2}{*}{ Bajo } & Recuento & 4 & 3 & 7 & 7 & 5 & 6 \\
\hline & $\%$ del total & 6.3 & 4.8 & 11.1 & 11.1 & 7.9 & 9.5 \\
\hline \multirow{2}{*}{$<$ Término medio } & Recuento & 12 & 9 & 21 & 12 & 20 & 12 \\
\hline & $\%$ del total & 19.0 & 14.3 & 33.3 & 19.0 & 31.7 & 19.0 \\
\hline \multirow{2}{*}{$>$ Término medio } & Recuento & 14 & 10 & 4 & 5 & 6 & 3 \\
\hline & $\%$ del total & 22.2 & 15.9 & 6.3 & 7.9 & 9.5 & 4.8 \\
\hline \multirow{2}{*}{ Alto } & Recuento & 2 & 5 & 3 & 3 & 5 & 5 \\
\hline & $\%$ del total & 3.2 & 7.9 & 4.8 & 4.8 & 7.9 & 7.9 \\
\hline \multirow{2}{*}{ Muy alto } & Recuento & 4 & 0 & 1 & 0 & 0 & 1 \\
\hline & $\%$ del total & 6.3 & 0.0 & 1.6 & 0.0 & 0.0 & 1.6 \\
\hline \multirow{2}{*}{ Total } & Recuento & 36 & 27 & 36 & 27 & 36 & 27 \\
\hline & $\%$ del total & 57.1 & 42.9 & 57.1 & 42.9 & 57.1 & 42.9 \\
\hline
\end{tabular}


Tabla 3. Factores psicosomáticos asociados al estrés.

\begin{tabular}{|c|c|c|c|c|c|c|c|}
\hline & \multicolumn{2}{|c|}{ Trastornos del sueño } & \multicolumn{2}{|c|}{ Trastornos gástricos } & \multicolumn{2}{|c|}{ Trastornos neuróticos } \\
\hline & & Casados & Solteros & Casados & Solteros & Casados & Solteros \\
\hline \multirow{2}{*}{ Bajo } & Recuento & 8 & 4 & 0 & 1 & 0 & 1 \\
\hline & $\%$ del total & 12.7 & 6.3 & 0.0 & 1.6 & 0.0 & 1.6 \\
\hline \multirow{2}{*}{$<$ Término medio } & Recuento & 9 & 12 & 13 & 8 & 26 & 17 \\
\hline & $\%$ del total & 14.3 & 19.0 & 20.6 & 12.7 & 41.3 & 27.0 \\
\hline \multirow{2}{*}{ > Término medio } & Recuento & 8 & 5 & 7 & 8 & 6 & 4 \\
\hline & $\%$ del total & 12.7 & 7.9 & 11.1 & 12.7 & 9.5 & 6.3 \\
\hline \multirow{2}{*}{ Alto } & Recuento & 7 & 3 & 5 & 1 & 2 & 3 \\
\hline & $\%$ del total & 11.1 & 4.8 & 7.9 & 1.6 & 3.2 & 4.8 \\
\hline \multirow{2}{*}{ Muy alto } & Recuento & 4 & 3 & 11 & 9 & 2 & 2 \\
\hline & $\%$ del total & 6.3 & 4.8 & 17.5 & 14.3 & 3.2 & 3.2 \\
\hline \multirow{2}{*}{ Total } & Recuento & 36 & 27 & 36 & 27 & 36 & 27 \\
\hline & $\%$ del total & 57.1 & 42.9 & 57.1 & 42.9 & 57.1 & 42.9 \\
\hline & & \multicolumn{2}{|c|}{ Trastornos por dolor } & \multicolumn{2}{|c|}{ Trastornos de ansiedad } & \multicolumn{2}{|c|}{ Trastornos psicosexuales } \\
\hline & & Casados & Solteros & Casados & Solteros & Casados & Solteros \\
\hline \multirow{2}{*}{ Bajo } & Recuento & 3 & 1 & 0 & 2 & 0 & 1 \\
\hline & $\%$ del total & 4.8 & 1.6 & 0.0 & 3.2 & 0.0 & 1.6 \\
\hline \multirow{2}{*}{$<$ Término medio } & Recuento & 14 & 11 & 22 & 12 & 21 & 17 \\
\hline & $\%$ del total & 22.2 & 17.5 & 34.9 & 19.0 & 33.3 & 27.0 \\
\hline \multirow{2}{*}{$>$ Término medio } & Recuento & 8 & 6 & 3 & 6 & 7 & 2 \\
\hline & $\%$ del total & 12.7 & 9.5 & 4.8 & 9.5 & 11.1 & 3.2 \\
\hline \multirow{2}{*}{ Alto } & Recuento & 5 & 5 & 4 & 0 & 3 & 4 \\
\hline & $\%$ del total & 7.9 & 7.9 & 6.3 & 0.0 & 4.8 & 6.3 \\
\hline \multirow{2}{*}{ Muy alto } & Recuento & 6 & 4 & 7 & 7 & 5 & 3 \\
\hline & $\%$ del total & 9.5 & 6.3 & 11.1 & 11.1 & 7.9 & 4.8 \\
\hline \multirow{2}{*}{ Total } & Recuento & 36 & 27 & 36 & 27 & 36 & 27 \\
\hline & $\%$ del total & 57.1 & 42.9 & 57.1 & 42.9 & 57.1 & 42.9 \\
\hline & & \multicolumn{2}{|c|}{ Trastornos depresivos } & & & & \\
\hline & & Casados & Solteros & & & & \\
\hline \multirow{2}{*}{ Bajo } & Recuento & 16 & 10 & & & & \\
\hline & $\%$ del total & 25.4 & 15.9 & & & & \\
\hline \multirow{2}{*}{$<$ Término medio } & \begin{tabular}{|l|} 
Recuento \\
\end{tabular} & 10 & 7 & & & & \\
\hline & $\%$ del total & 15.9 & 11.1 & & & & \\
\hline \multirow{2}{*}{ > Término medio } & Recuento & 5 & 4 & & & & \\
\hline & $\%$ del total & 7.9 & 6.3 & & & & \\
\hline \multirow{2}{*}{ Alto } & Recuento & 3 & 3 & & & & \\
\hline & $\%$ del total & 4.8 & 4.8 & & & & \\
\hline \multirow{2}{*}{ Muy alto } & Recuento & 2 & 3 & & & & \\
\hline & $\%$ del total & 3.2 & 4.8 & & & & \\
\hline Total & Recuento & 36 & 27 & & & & \\
\hline Iotal & $\%$ del total & 57.1 & 42.9 & & & & \\
\hline
\end{tabular}


El agotamiento puede provocar afectaciones psíquicas, como el desarrollo de la ansiedad o la depresión (Uribe, 2010). Según los resultados, fue la tendencia que se evidencia con mayor amplitud en el total de los sujetos, lo que permite inferir que las afecciones físicas y emocionales son una constante que se deriva de las circunstancias estresantes en quienes se encuentran en un relación de pareja y predispone a conductas que limitan la capacidad de retención de los participantes en su quehacer académico y laboral.

No obstante, debe resaltarse que aunque no manifestaron grandes complicaciones en cuanto a despersonalización e insatisfacción de logro, mostraron una potencial propensión a sufrir estrés crónico, gestado por el tiempo en pareja a causa de los horarios extendidos, las actividades propias del posgrado y las responsabilidades inherentes a su ejercicio profesional, considerando además las propias relacionadas con la personalidad del participante.

A partir de lo anteriormente planteado, y con la finalidad de equiparar la población de estudiantes (con pareja o sin pareja) que se hallaba en mayor riesgo de desarrollar alguno de los factores psicosomáticos asociados al estrés, en la Tabla 3 se muestra el grado de afectación en cada factor (bajo, abajo del término medio $(<)$, arriba del término medio $(>)$, alto y muy alto).

Como se puede observar en la tabla anterior, los participantes casados fueron más propensos a desarrollar trastornos del sueño, pues en su gran mayoría se ubicaron por arriba del término medio, lo que implica agotamiento por no conciliar adecuadamente el sueño. De la misma manera, presentaron una mayor persistencia de trastornos gastrointestinales que ponían en riesgo su salud fisiológica; así, de manera recurrente sufrían malestares gastrointestinales, como gastritis, úlceras $\mathrm{u}$ otros padecimientos asociados a esta patología.

Al contrario de los resultados anteriores, se puede observar que quienes padecían con mayor frecuencia los trastornos neuróticos fueron los estudiantes que no tenían una relación de pareja; aunque las cifras de incidencia no fueron altas, al menos $6.3 \%$ de ellos sufrían de este padecimiento pues se encontraban en niveles altos, y $3.2 \%$ en un nivel muy alto.
Los trastornos de dolor generan sintomatologías que pueden provocar alteraciones fisiológicas, mentales y comportamentales. Los estudiantes que mantenían una relación fueron los más propensos a sufrir esta condición, pues $12.7 \%$ alcanzó niveles por arriba del término medio, y 9.5\% niveles muy altos, causando el consumo de analgésicos para evitar o aliviar esta sintomatología, que suele alterar de manera considerable el comportamiento de quien la padece.

En cuanto al desglose de los trastornos de ansiedad, en ambas poblaciones el indicador fue muy alto (11.1\%), de modo que el desarrollo de este trastorno es independiente de tener o no una relación de pareja, pero que se desarrolla por la sobrecarga laboral que se experimenta.

En cuanto a la vida sexual, quienes tenían una relación de pareja fueron los más propensos a desarrollar trastornos psicosexuales debidos a la sobrecarga laboral; en $11.1 \%$ de estos se ubicó en el indicador por arriba del término medio, y en $7.9 \%$ alcanzó índices muy altos, lo que ponía en riesgo su relación.

Finalmente, en $4.8 \%$ de los participantes los trastornos depresivos se ubicaron en el nivel muy alto, más que en quienes no tenían ninguna relación de pareja; lo anterior puede deberse a que se sentían solos o que no podían comunicar a alguna persona de su confianza la situación en la que se hallaban, resultado de la sobrecarga académica y laboral.

\section{DISCUSIÓN}

El presente estudio identifica el grado de afectación que el estrés -considerado como uno de los problemas más estudiados en los últimos tiempos en razón de los trastornos que puede generar continuamente en quienes lo padecen- había tenido en los estudiantes de posgrado de un centro de estudios especializados por la sobrecarga sufrida en las actividades que desempeñaban y, con ello, el desarrollo de trastornos diversos.

Con la finalidad de identificar qué tipo de estudiantes muestran una mayor recurrencia de síntomas asociados al estrés y al desarrollo de distintos padecimientos, la muestra de estudio fue dividida en dos grupos: estudiantes que mantenían una relación de pareja y aquellos sin relación alguna. 
Los resultados muestran que había afectaciones significativas en los primeros, traducida en un agotamiento continuo, quizás originado por trastornos del sueño, pues se encontró que este factor psicosomático es el que con mayor frecuencia sufría este grupo; además de experimentar una sobrecarga de actividades académicas, también debían cubrir diversas exigencias laborales y familiares.

Derivado de los resultados obtenidos, se debe considerar como una medida importante establecer en las instituciones académicas de posgrado departamentos orientados a atender este tipo de padecimientos - mismos que pueden afectar de manera considerable el estado de salud de los propios estudiantes- mediante estrategias que permitan reducir los grados de estrés que comúnmente se suelen generar en esta población, tal como el estudio lo refleja, para así contribuir a mejorar su salud por medio de un desarrollo integral que haga posible, además de su enriquecimiento profesional, la adquisición de habilidades y recursos que canalicen de una mejor manera el estrés que dichas actividades ocasionan, evitando así la recurrencia de trastornos psicosomáticos.

Ante ello, el profesional de la salud mental debe ser un pilar indispensable en este tipo de instituciones, pues es quien puede propiciar una mayor estabilidad emocional en quienes deciden incursionar en estudios de posgrado, propiciando, además de la adquisición de los conocimientos académicos requeridos, el sano desarrollo psícológico de los educandos.

\section{REFERENCIAS}

Andere M., E. (2012). Pedagógica y los maestros del siglo XXI. Educación 2001, 24-25. Recuperado de http://www.eduardoandere.net/articulos/revista-educacion-2001/junio2012.pdf.

Ayuso M., J.A. (2006). Profesión docente y estrés laboral: una aproximación a los conceptos de estrés laboral y burnout. Revista Iberoamericana de Educación, 39(3), 1-15.

Barraza, A. (2008). El estrés académico en alumnos de maestría y sus variables moduladores: en diseño de diferencias de grupo. Avances en Psicología Latinoamericana, 26(2), 270-289.

Berrio, N. y Mazo, R. (2011). Estrés académico. Revista de Psicología de la Universidad de Antioquia, 3(2), 65-82.

Berumen Q., L. y González R., M. (2011). Relación entre depresión, estrés y estresores en adolescentes gestantes bajo distintas condiciones de estado civil. Summa Psicológica, 8, 32-38. doi: 10.18774/448x.2011.8.85.

Chiavenato, I. (2009). Comportamiento organizacional. México: McGraw-Hill.

Cólica P., R. (2012). Estrés, lo que usted quiere preguntar y saber. Córdoba (Argentina): Brujas.

El-Sahili, L. (2011). Docencia: riesgos y desafios. México: Trillas.

Golembiewski, R. y Munzenrider, R. (1984). Active and passive reactions to psychological burnout: Toward greater specificity in a phase model. Journal of Health and Human Resources Administration, 7, 264-268.

Gómez, J. (2014). Psicología de la sexualidad. Madrid: Alianza Editorial.

Hernández, Y. (2018). Nuevo rol del docente del siglo XXI. Gestiópolis. Recuperado de https://www.gestiopolis.com/nuevo-roldel-docente-del-siglo-xxi/.

Hernández S., R., Fernández C., C. y Baptista L., M.P. (2010). Metodología de la investigación (5ª ed.). México: McGraw-Hill.

Herrera, D., Coria, G.A., Muñoz, D.A., Graillet, O., Aranda, G.E., Rojas, F., Hernández, M. A. e Ismail, I.N. (2017). Impacto del estrés psicosocial en la salud. Neurobiología, 8(17), 1-23.

Leiter, M.P. y Maslach, C. (1988): The impact of interpersonal enviroment on burnout and organizational commitment. Journal of Occupational Behavior, 9, 297-308.

Maslach, C. (1976). Burn-out. Human Behaviour, 5(9), 16-22.

Maslach, C. y Jackson, S.E. (1982): Burnout in health professions: A social psychological analysis. En G. Sanders y J.Suls, (Eds.): Social psychology of health and illness (pp. 227-251). Hillsdale, NJ: Erlbaum.

Mayor M., C., Coral, V. y Ríos, J.R. (2009). Prevalencia de estrés, ansiedad y depresión en estudiantes de postgrado. Neurología, Neurocirugía y Psiquiatría, 42(1-4), 7-12.

Mazo, R., Londoño, K. y Gutiérrez Y., F. (2013). Niveles de estrés académico en estudiantes universitarios. Informes Psicológi$\cos , 13(2), 121-134$.

Montaño, J.M., Dossman, X., Herrera, J.A. Bronet, A. y Moreno, C.H. (2006). Helicobacter pylori y estrés psicosocial en pacientes con gastritis crónica. Colombia Médica, 37(2), 39-44. 
Naranjo M., L. (2009). Una revisión teórica sobre el estrés y algunos aspectos relevantes en este en el ámbito educativo. Educación, 33(2), 171-190.

Portilla D., R. (2006). Estrés y sueño. Revista Mexicana de Neurociencias, 7(1), 15-20. Recuperado de http://www.medigraphic. com/pdfs/revmexneu/rmn-2006/rmn061d.pdf .

Pulido R., M.A., Serrano, M.L., Valdez, E., Chávez, M.T., Hidalgo, P. y Vera, F. (2011). Estrés académico en estudiantes universitarios. Psicología y Salud, 13(1), 31-37.

Reyes L., V., Ibarra, D., Torres M., E.R. y Razo R., S. (2012). El estrés como un factor de riesgo en la salud: análisis diferencial entre docentes de universidades públicas y privadas. Revista Digital Universitaria, 13(7), 1-14. Recuperado de http://www. revista.unam.mx/vol.13/num7/art78/.

Sánchez P., T., Sirera, R., Peiró, G. y Palmero, F. (2008). Estrés, depresión, inflamación y dolor. Revista Electrónica de Motivación y Emoción, 11(26), 1-15. Recuperado de http://reme.uji.es/articulos/numero28/article1/article1.pdf.

Sarudiansky, M. (2013). Ansiedad, angustia y neurosis. Antecedentes conceptuales e históricos. Psicología Iberoamericana, 21(2), 19-28.

Secretaría de Educación Pública (2016). Estrategia Nacional de Formación Continua de Profesores de Educación Básica y Media Superior. México: SEP. Recuperado de https://www.gob.mx/sep/articulos/estrategia-nacional-de-formacion-continua-de-profesores-de-educacion-basica-y-media-superior-22969.

Selye, H. (1956). The stress of life. New York: McGraw-Hill.

Suárez, N. y Díaz, L.B. (2015). Estrés académico, deserción y estrategias de retención de estudiantes en la educación superior. Revista de Salud Pública, 17(2), 300-3013.

Toribio, C. y Franco, S. (2016). Estrés académico: el enemigo silencioso del estudiante. Salud y Administración, 3(7), 11-18.

Uribe, J. (2010). EDO: Escala de Desgaste Ocupacional (Burnout). México: El Manual Moderno, S.A.

Vieco G., F. y Abello, R. (2014). Factores psicosomáticos de origen laboral, estrés y morbilidad en el mundo. Psicología desde el Caribe, 31(2), 354-385.

Zúñiga R., M. (2014). El trastorno de ansiedad y estrés escolar en los adolescentes con alto desempeño académico. International Journal of Developmental and Educational Psychology, 2(1), 205-212. 